\title{
Highly stable charge generation layers using caesium phosphate as $n$-dopants and inserting interlayers
}

Cite as: J. Appl. Phys. 111, 103107 (2012); https://doi.org/10.1063/1.4720064

Submitted: 02 February 2012 . Accepted: 19 April 2012 . Published Online: 22 May 2012

Carola Diez, Thilo C. G. Reusch, Erwin Lang, Thomas Dobbertin, and Wolfgang Brütting

\section{ARTICLES YOU MAY BE INTERESTED IN}

High power efficiency tandem organic light-emitting diodes based on bulk heterojunction organic bipolar charge generation layer

Applied Physics Letters 98, 243309 (2011); https://doi.org/10.1063/1.3599557

Impedance spectroscopy as a probe for the degradation of organic light-emitting diodes

Journal of Applied Physics 107, 054501 (2010); https://doi.org/10.1063/1.3294642

Power efficiency improvement in a tandem organic light-emitting diode

Applied Physics Letters 92, 223311 (2008); https://doi.org/10.1063/1.2938269

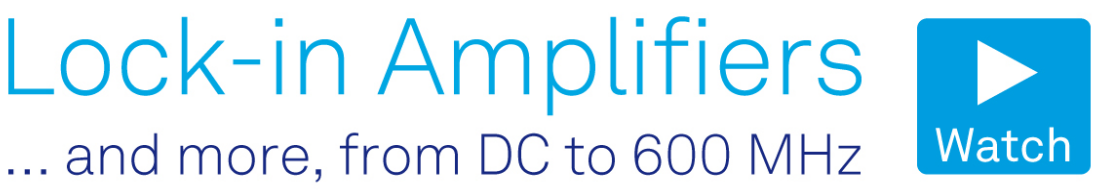




\title{
Highly stable charge generation layers using caesium phosphate as $\mathbf{n}$-dopants and inserting interlayers
}

\author{
Carola Diez, ${ }^{1,2, a)}$ Thilo C. G. Reusch, ${ }^{1}$ Erwin Lang, ${ }^{1}$ Thomas Dobbertin, ${ }^{1}$ \\ and Wolfgang Brütting ${ }^{2}$ \\ ${ }^{1}$ OSRAM Opto Semiconductors GmbH, Leibnizstrasse 4, 93055 Regensburg, Germany \\ ${ }^{2}$ Institute of Physics, University of Augsburg, Universitätsstrasse 1, 86159 Augsburg, Germany
}

(Received 2 February 2012; accepted 19 April 2012; published online 22 May 2012)

\begin{abstract}
Highly stable and efficient charge generation layers (CGLs) comprising caesium phosphate $\left(\mathrm{Cs}_{3} \mathrm{PO}_{4}\right)$ doped 2,9-dimethyl-4,7-diphenyl-1,10-phenanthroline (BCP) as $n$-type organic semiconductor and molybdenum trioxide $\left(\mathrm{MoO}_{3}\right)$ doped $\mathrm{N}, \mathrm{N}^{\prime}$-di-(naphthalen-1-yl)-N, $\mathrm{N}^{\prime}$-diphenyl-benzidine $(\alpha-\mathrm{NPD})$ as $p$-type organic semiconductor, respectively, are presented. By inserting narrow-gap organic copper-phthalocyanine $(\mathrm{CuPc})$ and wide-gap insulating aluminum oxide $\left(\mathrm{Al}_{2} \mathrm{O}_{3}\right)$ as interlayer (IL), we show that the long-term stability of the CGL can be improved. The variation of the $\mathrm{CuPc}$ IL thickness yields an optimum of $8 \mathrm{~nm}$ as a trade-off between minimal operating voltage and maximum voltage stability of the CGL. Luminance-current density-voltage characteristics and lifetime measurements of stacked green organic light emitting diodes (OLEDs) confirm the functionality and high voltage stability of the presented CGL. The luminous efficacy of the stacked OLED compared to the non-stacked reference device is nearly unchanged. However, the lifetime of the stacked device is enhanced by a factor of 3.5. Consistent with our experimental findings, we propose a model of the energy-level diagram of a fully doped CGL with IL based on a field-assisted tunneling mechanism. (C) 2012 American Institute of Physics. [http://dx.doi.org/10.1063/1.4720064]
\end{abstract}

\section{INTRODUCTION}

Organic light emitting diodes (OLEDs) have attracted increasing attention in the recent years, since they are promising candidates for high-efficiency solid state light sources. ${ }^{1} \mathrm{~A}$ long operation lifetime of the OLED devices without reduction in luminous flux has to be ensured to increase their acceptance in the growing market of new lighting technologies and allow for mass-production. One elegant way to improve the lifetime is vertical stacking of a number of OLEDs on top of each other. ${ }^{2-5}$ This stacking approach reduces the electrical stress for each individual OLED unit, resulting in an enhanced lifetime at a given luminance level. The connecting element of the individual OLED units is commonly called a charge generation layer (CGL). ${ }^{2}$ Since the first studies by Kido et al. ${ }^{2}$ using indium tin oxide (ITO) or ( $\mathrm{F}_{4}$-TCNQ) adjacent to a hole transport layer (HTL) as CGLs, many other materials were investigated. ${ }^{3,6-12}$ Under suitable bias conditions, the connection unit should be capable of generating electron-hole pairs, separating them and supplying these charges to the neighboring functional layers. The main requirement is that the charge generation takes place with a lowest possible voltage drop across the CGL. This means that a two-fold stacked OLED roughly consumes twice the voltage of a non-stacked OLED. The most crucial point for lighting applications is the reduction of voltage increase during operation of the stacked OLED, and hence the charge generation layer, under constant current conditions.

Charge generation layers based on thin metal films, transparent conductive oxides and transition metal oxides

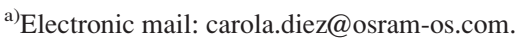

(TMOs) have been demonstrated. ${ }^{3,10-12}$ Another approach is using a doped organic $p-n$ heterojunction as suggested by Liao et $a l .{ }^{6}$ The latter type can be described by a fieldinduced charge-carrier separation at the doped organic/ organic heterointerface via tunneling of electrons through the depletion zone from the highest occupied molecular orbital (HOMO) of the $p$-type layer to the lowest unoccupied molecular orbital (LUMO) of the $n$-type layer. ${ }^{13}$ The $p$-type dopant can either be organic such as $\mathrm{F}_{4}$-TCNQ or a transparent metal oxide, like $\mathrm{WO}_{3}, \mathrm{~V}_{2} \mathrm{O}_{5}$ or molybdenum trioxide $\left(\mathrm{MoO}_{3}\right)$ as used in this work. ${ }^{6,9,13}$ Elementary alkaline metals such as Li, $\mathrm{Cs}$, and $\mathrm{Mg}$ or alkaline metal complexes as caesium carbonate $\left(\mathrm{Cs}_{2} \mathrm{CO}_{3}\right)$ are typically used for doping of electron transport layers (ETLs). ${ }^{6-8,12-15}$

For future mass-production, it is essential that the used organic materials are easily processable and cost efficient. Very recently, Wemken et al. demonstrated that caesium phosphate $\left(\mathrm{Cs}_{3} \mathrm{PO}_{4}\right)$ is both a low cost and efficient $n$-type dopant in OLEDs. ${ }^{16}$

To the best of our knowledge, we are the first to demonstrate that $\mathrm{Cs}_{3} \mathrm{PO}_{4}$ can also be used in CGLs with improved performance, e.g., regarding drive voltage. Furthermore, this material overcomes some of the difficulties encountered with other $n$-type dopants, such as the need of special evaporation cells, absorption in the visible range, migration of dopants and hence instability of the fabricated devices. ${ }^{17}$

So far, there are only few reports on long-term stability of stacked OLEDs or charge generation layers under real operation conditions. ${ }^{5}$ In this paper, we address this issue in a detailed and systematic study and show that by inserting both a narrow-gap organic material and a wide-gap oxidic material as interlayer (IL) between the $p$-doped and the 
$n$-doped part, the operating voltage can be minimized under constant current conditions and the long-term stability of the devices can be improved. Consistent with our experimental findings we propose a model of an energy-level diagram for a fully doped CGL with IL based on a field-assisted tunneling mechanism.

This paper is organized as follows: The experimental details of the fabricated CGL test devices and the stacked green light-emitting diodes are given in Sec. II. Section III A shows the influence of an organic interlayer within the doped $p$ - $n$ heterojunction as compared to a wide-gap oxidic interlayer. The results of the stacked green OLEDs, including efficacies and lifetime are presented in Sec. III B. The paper concludes with a discussion about the physical mechanism of an interlayer within a doped $p-n$ junction (Sec. IV).

\section{EXPERIMENTAL DETAILS}

All devices prepared in this study exhibited an active area of $4 \mathrm{~mm}^{2}$ and were fabricated in a vacuum deposition chamber at a base pressure of $10^{-7}$ mbar. Before evaporation the patterned ITO glass substrates were cleaned with solvents in a multistep process and exposed to oxygen plasma. Before loading into the evaporation cell, the $\mathrm{Cs}_{3} \mathrm{PO}_{4}$ powder was dried at $440^{\circ} \mathrm{C}$ for over $6 \mathrm{~h}$ under inert atmosphere to remove traces of water. All other materials were used as received. The rate of deposition for the organic materials was $0.05 \mathrm{~nm} / \mathrm{s}$, and the substrates were not heated during evaporation. The doped layers were directly formed from the gas phase by co-evaporation of the host material and the dopant. This deposition technique was also used for the green dye inside the host. Prior to testing, the devices were encapsulated under inert gas atmosphere with a glass lid and getter containing zeolite as dryer. Current density-voltage characteristics and electroluminescence (EL) spectra were measured using a source measurement unit (Keithley 2400-C) and a spectrometer (Instrument Systems CAS 140 with TOP100). The luminance over time was detected by a blue enhanced silicon photodiode (Photonic Detectors, Inc., PDB-C613) under constant current conditions.

\section{A. CGL test devices}

For a fast investigation and optimization of charge generation layers, a simplified test structure was developed. The general layer sequence is shown in Fig. 1(a), including thicknesses. The doping concentration of both $\mathrm{Cs}_{3} \mathrm{PO}_{4}$ and $\mathrm{MoO}_{3}$ was $10 \%$.

The test structure is designed in such a way that by applying a positive bias to the ITO anode a tunneling current can be measured (see Fig. 1(b)). This polarity for the CGL is the same as in the stacked OLED. The undoped 2,9-dimethyl-4,7diphenyl-1,10-phenanthroline (BCP) and N,N'-Di-(naphthalen-1-yl)-N, $N^{\prime}$-diphenyl-benzidine $(\alpha-N P D)$ layers prevent direct charge carrier injection from the electrodes under these bias conditions to ensure that the measured current results from charge generation at the $p-n$ junction. Two different types of interlayer materials were used. The first device set comprising the narrow-gap organic material copperphthalocyanine $(\mathrm{CuPc})$ and the second device set comprising

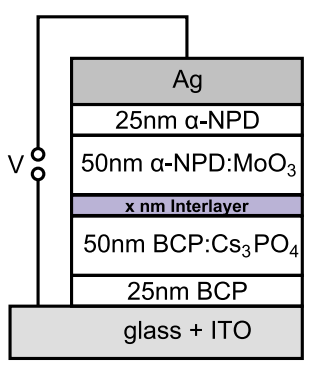

(a.) CGL test device

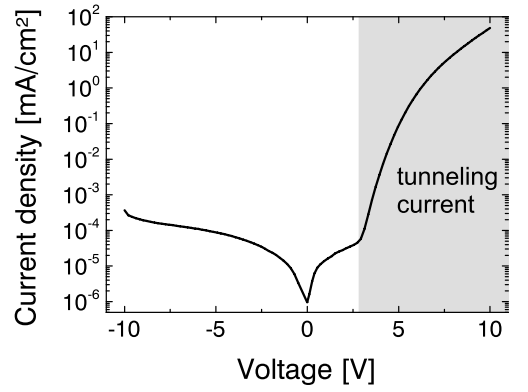

(b.) $\mathrm{j}-\mathrm{V}$ characteristic
FIG. 1. (a.) Device structure of the CGL test device with different IL thickness $x$. The doping concentration of both $\mathrm{Cs}_{3} \mathrm{PO}_{4}$ and $\mathrm{MoO}_{3}$ was $10 \%$. (b.) Exemplary j-V characteristics of a CGL test device.

the wide-gap insulator material aluminum oxide $\left(\mathrm{Al}_{2} \mathrm{O}_{3}\right)$. For the growth of the $\mathrm{Al}_{2} \mathrm{O}_{3}$ layers, a non-reactive $\mathrm{RF}$ magnetron sputtering process with a frequency of $13.56 \mathrm{MHz}$ was applied. The power density on the $\mathrm{Al}_{2} \mathrm{O}_{3}$ target was $2.3 \mathrm{~W} / \mathrm{cm}^{2}$ and the base pressure was about $3 \mu$ bars. The process gas was argon with a total flux of $70 \mathrm{sccm}$. All experiments were done without breaking vacuum between the organic deposition processes. The IL thickness of both series was varied between $0 \mathrm{~nm}$ and $16 \mathrm{~nm}$ in steps of $2 \mathrm{~nm}$. By a combinatorial evaporation and sputtering process with different shadow masks, it was possible to process the devices on the same substrate successively to ensure comparability of different devices.

\section{B. Stacked green light-emitting diodes}

To find the optimal position of the emitting layers (EMLs) of the green devices, optical simulations based on a dipole model were carried out. ${ }^{18-20}$ For comparison, a nonstacked reference device with the same organic layer thickness as the stacked devices was prepared. This is necessary to ensure an approximately equal mode distribution especially for ITO/organic-modes and therefore reduces influence of the optical microcavities. A schematic sketch of the nonstacked and stacked devices can be found in Fig. 2 .

The single $p-i$ - $n$ OLED structure consisted of an ITO anode, $5 \%$ molybdenum trioxide $\left(\mathrm{MoO}_{3}\right)$ doped $\alpha$-NPD for hole injection and transport $(180 \mathrm{~nm})$, undoped $\alpha$-NPD $(10 \mathrm{~nm})$ as electron blocking layer, $15 \%$ of a green phosphorescent dye fac-tris(2-phenly-pyridin)iridium $\left(\operatorname{Ir}(\mathrm{ppy})_{3}\right)$ in a predominantly electron conductive host (TMM004 (Ref. 21) as EML (10 nm), $10 \mathrm{~nm}$ thick TMM004 as hole blocking layer, 2\% $\mathrm{Cs}_{3} \mathrm{PO}_{4}$ doped $\mathrm{BCP}$ as an electron transport and injection layer $(60 \mathrm{~nm})$ and a $150 \mathrm{~nm}$ thick silver cathode. After that, we fabricated stacked $p-i-n$ OLEDs by simply stacking the single $p-i-n$ cell without or with an $8 \mathrm{~nm}$ thick CuPc IL within the $p$ - $n$ junction interconnection unit as shown in Fig. 2(b).

\section{RESULTS}

\section{A. CGL test devices}

To investigate the influence of a narrow-gap organic IL, namely, $\mathrm{CuPc}$, and a wide-gap insulating $\mathrm{IL}$, namely, $\mathrm{Al}_{2} \mathrm{O}_{3}$, within the $p-n$ junction, a series of CGL test devices with 


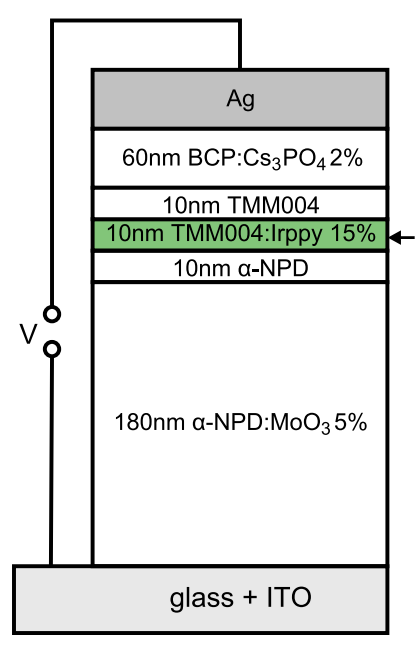

(a.) reference OLED

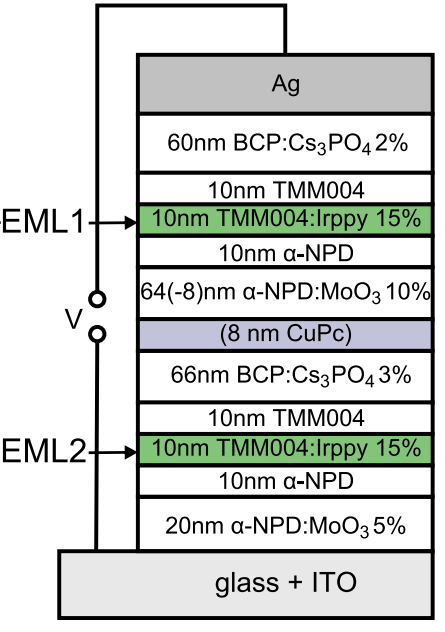

(b.) stacked OLED
FIG. 2. Device structure of the non-stacked (a) reference OLED and the stacked (b) OLED without and with an $8 \mathrm{~nm} \mathrm{CuPc} \mathrm{IL.} \mathrm{The} \mathrm{total} \mathrm{organic}$ layer thickness of all 3 fabricated devices is the same.

varying IL thickness were processed as described in Sec. II A. For comparison a reference device without IL was fabricated as well. Fig. 3 shows the $\mathrm{j}-\mathrm{V}$ characteristics.

It is remarkable that the steepest characteristic is measured for the device with $4 \mathrm{~nm} \mathrm{CuPc}$ IL and not for the reference device without IL. One would assume intuitively that by inserting an undoped IL material, the IL should act as a resistor and therefore the voltage drop across the CGL should be increased. But this is not the case here. The voltage drop across the CGL can even be reduced by inserting $4 \mathrm{~nm}$ of the organic IL CuPc. Whereas inserting $4 \mathrm{~nm} \mathrm{Al}_{2} \mathrm{O}_{3}$ leads to a rise of the applied voltage for a given tunneling current. The larger the $\mathrm{Al}_{2} \mathrm{O}_{3}$ layer thickness the flatter the $\mathrm{j}$ $\mathrm{V}$ characteristics become. It can be concluded that all CGL test devices comprising the $\mathrm{Al}_{2} \mathrm{O}_{3}$ IL have lower conductivity compared to the device without IL and the devices with $\mathrm{CuPc}$ IL, respectively. Therefore, an $\mathrm{Al}_{2} \mathrm{O}_{3} \mathrm{IL}$ acts as a carrier blocking layer in a doped $p-n$ junction.

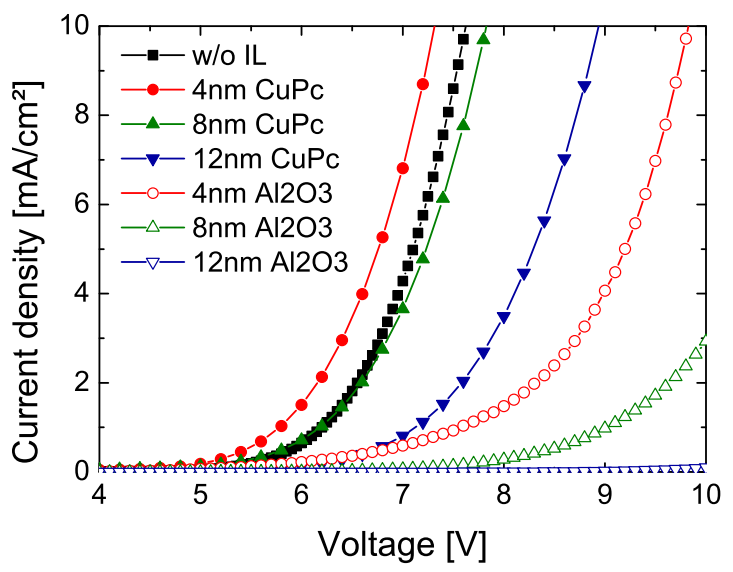

FIG. 3. Current density-voltage characteristics of CGL test devices (structure shown in Fig. 1) with a narrow-gap organic and wide-gap oxidic interlayer material of different thicknesses. (Filled symbols: $\mathrm{CuPc}$, open symbols: $\mathrm{Al}_{2} \mathrm{O}_{3}$ ).

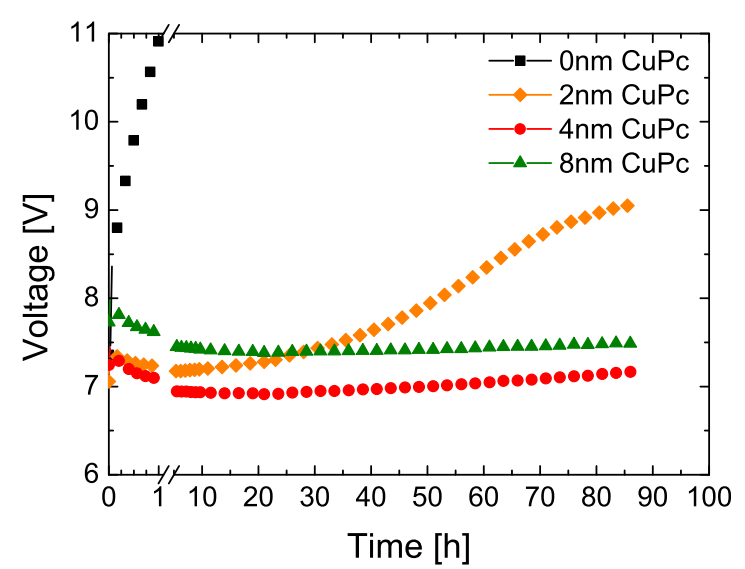

FIG. 4. Voltage dependency over time at a constant current density of $10 \mathrm{~mA} / \mathrm{cm}^{2}$ of CGL test devices. The CuPc IL thickness was varied between $0 \mathrm{~nm}$ and $8 \mathrm{~nm}$.

The stability of the CGL test device comprising the $\mathrm{CuPc}$ and the $\mathrm{Al}_{2} \mathrm{O}_{3}$ IL was investigated by applying a constant current density of $10 \mathrm{~mA} / \mathrm{cm}^{2}$ and monitoring the voltage over time. The experimental data can be found in Figs. 4 and 5, respectively. We note that these current densities correspond to accelerated testing conditions in OLEDs. For a high operational stability in an OLED, it is required that the voltage remains constant over time. Within the first hour a pronounced voltage rise for the device without IL can be measured. Clearly, such a behavior would be detrimental for a stacked OLED. At the same time, the devices with the CuPc IL (see Fig. 4) show a voltage drop, which we attribute to a temperature enhanced conductivity of organic materials due to heating during the measurement. ${ }^{22}$

At later times, a significant voltage rise can be seen for the device with $2 \mathrm{~nm} \mathrm{CuPc}$ during electrical aging. Only a small voltage rise can be found for the device with $4 \mathrm{~nm}$ $\mathrm{CuPc}$, whereas there is no voltage rise for the device with $8 \mathrm{~nm} \mathrm{CuPc}$.

The devices with the $\mathrm{Al}_{2} \mathrm{O}_{3}$ IL show qualitatively a similar behavior during electrical aging. With rising interlayer thickness, the voltage stability of the devices is increased. But we have to point out that for an acceptable stability of

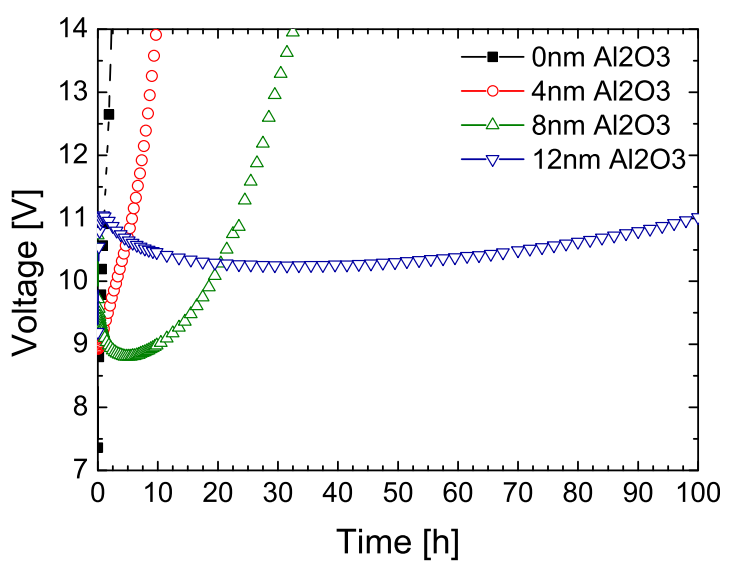

FIG. 5. Voltage dependency over time at a constant current density of $10 \mathrm{~mA} / \mathrm{cm}^{2}$ of CGL test devices. The $\mathrm{Al}_{2} \mathrm{O}_{3}$ IL thickness was varied between $0 \mathrm{~nm}$ and $12 \mathrm{~nm}$. 


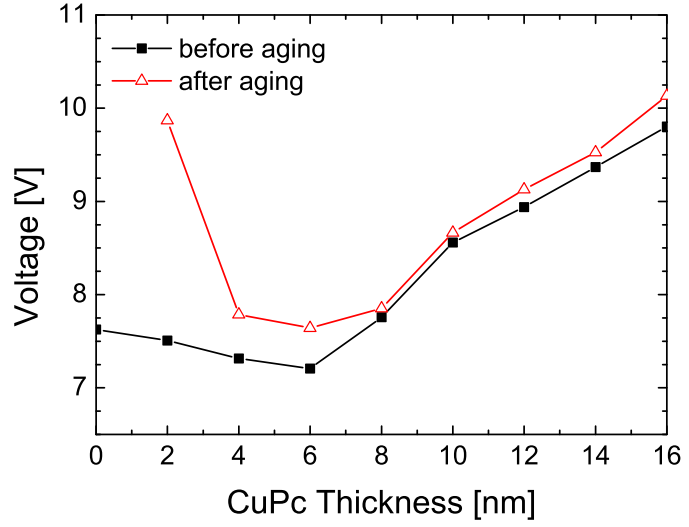

FIG. 6. Voltage dependency with respect to IL thickness at a constant current density of $10 \mathrm{~mA} / \mathrm{cm}^{2}$ of CGL test devices. Filled symbols: before electrical aging, open symbols: after electrical aging for $90 \mathrm{~h}$ at $10 \mathrm{~mA} / \mathrm{cm}^{2}$.

the CGL an $\mathrm{Al}_{2} \mathrm{O}_{3}$ IL thickness of at least $12 \mathrm{~nm}$ is needed. This finding is consistent with a proportionally high voltage drop across the CGL which would reduce the efficiency in a stacked OLED. Consequently, the devices with the CuPc IL and not with the $\mathrm{Al}_{2} \mathrm{O}_{3}$ IL are further examined.

To demonstrate the IL thickness dependency of the CGL stability, the voltage at a constant current density of $10 \mathrm{~mA} / \mathrm{cm}^{2}$ was extracted from the $\mathrm{j}-\mathrm{V}$ characteristics measurements for each CuPc layer thickness before and after electrical aging of the test devices, respectively. This serves as a figure of merit for the operating voltage of the CGL. Fig. 6 shows this dependency. As mentioned above, it can be seen clearly that there is a voltage minimum for a $\mathrm{CuPc}$ thickness of $4 \mathrm{~nm}$. With rising layer thickness the voltage drop at the CGL is increased. This holds true for devices before and after electrical aging. The lowest voltage rise can be found for devices with an IL thickness of $8 \mathrm{~nm}$ or larger. In conclusion, we observe an optimum of $8 \mathrm{~nm}$ CuPc thickness as a tradeoff between minimal voltage drop and maximum stability of the CGL.

\section{B. Stacked green light-emitting diodes}

Stacked green light-emitting diodes without and with an $8 \mathrm{~nm}$ thick $\mathrm{CuPc}$ IL as well as a non-stacked reference device with the same organic layer thickness were fabricated as described in Sec. II B. The current density-voltage characteristics and the luminance versus voltage of the three devices are presented in Fig. 7.

For a given current density, the stacked devices consume slightly more than twice the voltage of the non-stacked device. This can be explained by an equivalent circuit where the individual diodes, and for a stacked device the individual emission units, are connected in series. The remaining difference can be attributed to a small voltage drop across the CGL. At $1000 \mathrm{~cd} / \mathrm{m}^{2}$ the stacked OLED without IL exhibits an operation voltage of $6.3 \mathrm{~V}$, which is larger than twice the operation voltage $(2.7 \mathrm{~V})$ of the non-stacked reference OLED. However, the incorporation of the CuPc IL leads to a reduction in operation voltage to $5.9 \mathrm{~V}$ at $1000 \mathrm{~cd} / \mathrm{m}^{2}$, indicating that this effectively enhances the tunneling probability

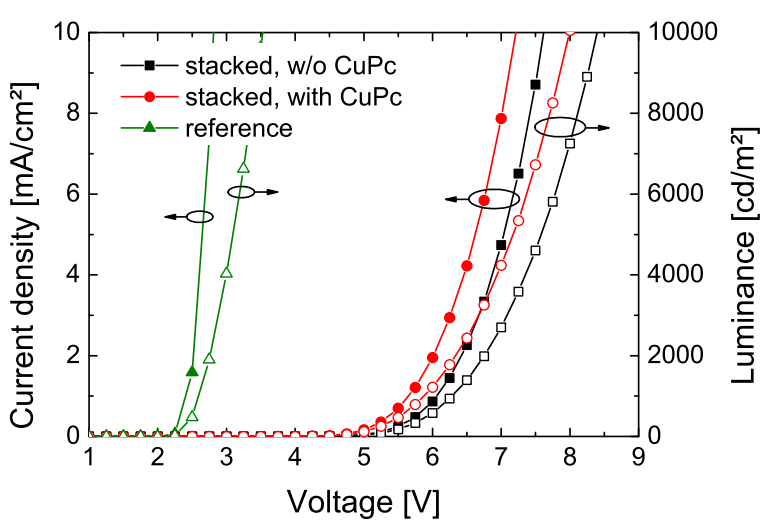

FIG. 7. Current density-voltage characteristics (filled symbols) and luminance (open symbols) of green stacked OLEDs and a non-stacked reference device. The stacked OLEDs were fabricated without and with an $8 \mathrm{~nm}$ thick CuPc interlayer.

of charge carriers through the depletion zone at the interface of the doped organic $p$ - $n$ junction. The additional $0.5 \mathrm{~V}$ voltage drop compared to twice the voltage of the non-stacked device can be attributed to the CGL. However, we have to point out that the CGL is working well, if one takes into account that the CGL has to overcome approximately $3 \mathrm{~V}$ built-in potential, which is the difference between the LUMO of BCP (Ref. 23) and the HOMO of $\alpha$-NPD. ${ }^{24}$ Few results with a comparable voltage drop across the CGL have been reported in the literature. ${ }^{5,14}$ One possibility to reduce this voltage drop further could be a tuning of the concentration of $\mathrm{Cs}_{3} \mathrm{PO}_{4}$ and $\mathrm{MoO}_{3}$ in the doped layers resulting in a better energy-level alignment at the interface.

Fig. 8 shows the normalized electroluminescence spectra of the stacked and non-stacked green OLEDs measured at $10 \mathrm{~mA} / \mathrm{cm}^{2}$. All three OLEDs exhibit the same EL intensity without any narrowing of the spectrum. The emission profile follows a Lambertian distribution (not shown here) for all three devices. These findings are a clear indication that the positioning of the EMLs within the microcavity was correct and that all devices are optically comparable.

The current efficiency as well as the luminous efficacy of the stacked and reference devices, respectively, are displayed in Fig. 9. The data were measured on devices without

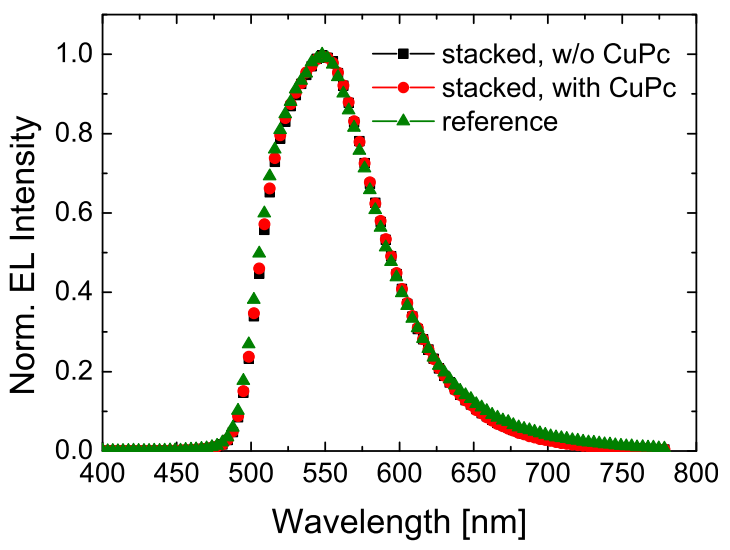

FIG. 8. Normalized emission spectra of stacked devices without and with $\mathrm{CuPc}$ interlayer as well as a non-stacked reference device. Measurements were done at a constant current density of $10 \mathrm{~mA} / \mathrm{cm}^{2}$. 


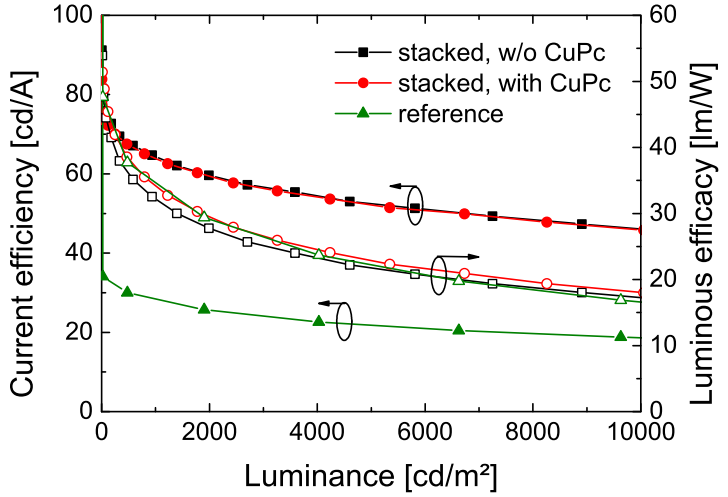

FIG. 9. Current efficiency (filled symbols) and luminous efficacy (open symbols) of stacked devices without and with $\mathrm{CuPc}$ interlayer with respect to a non-stacked reference device.

any outcoupling enhancement via half ball lenses, microlens arrays or scattering foils. ${ }^{20}$ At $1000 \mathrm{~cd} / \mathrm{m}^{2}$ the current efficiency of both stacked devices is $64 \mathrm{~cd} / \mathrm{A}$. Comparing with the non-stacked device $\left(28 \mathrm{~cd} / \mathrm{A}\right.$ at $\left.1000 \mathrm{~cd} / \mathrm{m}^{2}\right)$, the current efficiency of the stacked devices is more than doubled. This can be explained by an optical benefit of the second cavity maximum for EML2 (see Fig. 2) in the stacked device, where plasmonic losses are avoided. ${ }^{25}$ However, this gain in current efficiency is compensated by a slightly larger operating voltage of the CGL, resulting in comparable luminous efficacy of all three OLEDs $\left(34 \mathrm{~lm} / \mathrm{W}\right.$ at $\left.1000 \mathrm{~cd} / \mathrm{m}^{2}\right)$.

One of the most important parameters in real OLED applications is the lifetime of the devices, which goes hand in hand with the voltage stability. An accelerated lifetime measurement at an initial luminance of $4000 \mathrm{~cd} / \mathrm{m}^{2}$ under constant current conditions was performed. For the stacked and non-stacked devices, the constant current density was $j=7.5 \mathrm{~mA} / \mathrm{cm}^{2}$ and $j=18.7 \mathrm{~mA} / \mathrm{cm}^{2}$, respectively. The luminance decay and the voltage stability over the operating time are presented in Fig. 10. The time to $70 \%$ of the initial luminance $\left(\mathrm{LT}_{70}\right)$ for the non-stacked reference device is $180 \mathrm{~h}$, whereas for the stacked device without CuPc IL it is $580 \mathrm{~h}$. These numbers are outperformed by the lifetime of the stacked device with the CuPc IL, which is $630 \mathrm{~h}$. This means that the lifetime of the stacked device is 3.5 times

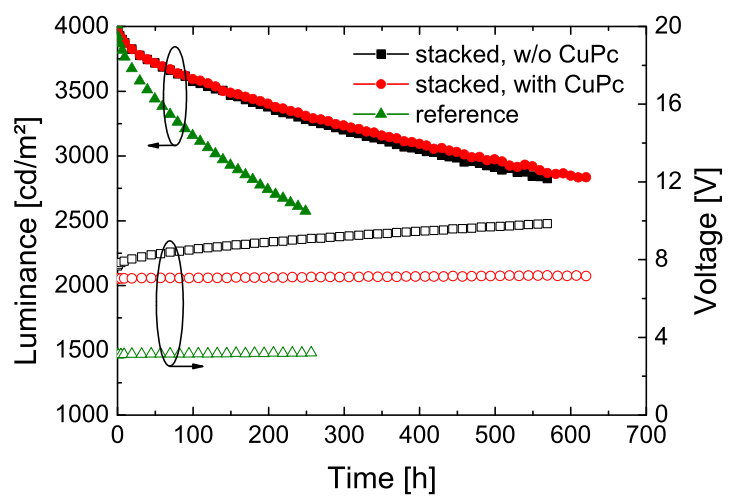

FIG. 10. Luminance (filled symbols) and voltage (open symbols) versus time of stacked devices without and with CuPc interlayer in comparison to a non-stacked reference device. Measurements were done at an initial luminance of $4000 \mathrm{~cd} / \mathrm{m}^{2}$. higher compared to the non-stacked device. The lifetime at a common initial luminance of $1000 \mathrm{~cd} / \mathrm{m}^{2}$ can easily be calculated from the measured data by using the well-known relationship $L_{0}^{n} \times \tau=$ const., ${ }^{26}$ with the initial luminance $L_{0}$, the acceleration factor $n$, and the measured lifetime $\tau$. With the lifetime measurement of the reference device at $4000 \mathrm{~cd} / \mathrm{m}^{2}$ and by approximating an equal luminance output from both emission units in the stacked device, i.e., each unit emits $2000 \mathrm{~cd} / \mathrm{m}^{2}$ leading to the lifetime of $630 \mathrm{~h}$, an acceleration factor of $n=1.8$ can be calculated. This results in an extrapolated lifetime of over $7500 \mathrm{~h}\left(\mathrm{LT}_{70}\right)$ at $1000 \mathrm{~cd} / \mathrm{m}^{2}$. Most remarkably, however, there is no voltage rise for the device with the $8 \mathrm{~nm}$ IL during operation. This clearly demonstrates the superior performance of the investigated CGL system consisting of caesium phosphate as a novel n-dopant and $\mathrm{CuPc}$ as interlayer.

\section{DISCUSSION}

The understanding of the working mechanisms of charge generation in different CGL architectures is an active field of research. ${ }^{3,5,13}$ Generally, CGL architectures are divided into two classes. ${ }^{3,13}$ In the first class, the CGL is formed by a doped $p-n$ heterojunction where the charge generation takes place at the interface between the $p$ - and $n$ doped layers. ${ }^{13}$ The second class comprises TMOs. In the latter case, charge generation takes place at the interface between the hole transporting material and the TMO. ${ }^{3,27}$ Gao et al. proposed an architecture where only a $p$-type doped organic layer together with $\mathrm{Al}_{2} \mathrm{O}_{3}$ as hole blocking layer serve as CGL. ${ }^{28}$ So far, the focus of research has been on minimizing the voltage drop across the CGL and thus maximizing the charge generation efficiency, but less attention has been paid to the long-term stability of CGLs. Our results show that the voltage stability of the CGL can be improved by inserting an IL. One hypothesis is that the IL prevents a chemical reaction at the interface between different species of the $n$ - and $p$-type doped layers or dopant interdiffusion. Both of these detrimental effects can lead to additional tunneling barriers and result in traps or compensation effects.

This assumption is supported by the observation that for both IL, the voltage rise during operation is decreased in comparison to CGLs without IL (see Figs. 4 and 5, respectively). CuPc is a well-known material which was often used for improving hole injection, ${ }^{29-31}$ whereas wide-gap insulating materials as $\mathrm{Li}_{2} \mathrm{O}$ or $\mathrm{Al}_{2} \mathrm{O}_{3}$ were often used for improving the electron injection ${ }^{32}$ by optimizing the band alignment. The advantage of $\mathrm{CuPc}$ is, that closed layers are formed at small thicknesses. ${ }^{29}$ In contrast, a larger thickness of $\mathrm{Al}_{2} \mathrm{O}_{3}$ compared to $\mathrm{CuPc}$ is needed, because of its different wetting behavior. We found in our measurements inhomogeneities for the $\mathrm{Al}_{2} \mathrm{O}_{3}$ layer thickness which can be attributed to the sputtering process (not shown here). Therefore, larger thicknesses are necessary to form closed layers with sputtered $\mathrm{Al}_{2} \mathrm{O}_{3}$. Once a closed layer is formed, the IL acts as a physical barrier between the reactive materials.

It is striking that we observe a decrease of the voltage drop across the CGL with increasing $\mathrm{CuPc}$ thickness within the first $4 \mathrm{~nm}$ (see Figs. 3 and 6). Intuitively, one would 
expect an additional voltage drop due to the undoped dielectric material acting as a resistor. In fact, this is what we observe with the $\mathrm{Al}_{2} \mathrm{O}_{3}$ IL, but not with CuPc. Instead, for $\mathrm{CuPc}$ the voltage drop decreases up to a layer thickness of 4-8 $\mathrm{nm}$, corresponding to the formation of a closed layer ${ }^{29-31}$ and thus resulting in a smaller barrier and hence higher tunneling current as observed in our experiments.

We now turn to the mechanism of charge generation. $\mathrm{Al}_{2} \mathrm{O}_{3}$ is a wide-gap insulating material, whereas $\mathrm{CuPc}$ is a material with a narrow-gap lying between the HOMO of the $p$-doped layer and the LUMO of the $n$-doped layer. ${ }^{23,33}$ Fig. 11 shows a simplified drawing of the CGL energy-level diagram for devices without IL ((a) and (b)), with $\mathrm{Al}_{2} \mathrm{O}_{3} \mathrm{IL}$ ((c) and (d)), and with CuPc IL ((e) and (f)). The energylevel diagram for the device without IL is taken from Kröger et al. Note that in an ideal $p$ - $n$ junction without considering interfacial dipoles the depletion zone is in the order of 10-20 nm assuming typical free carrier concentrations of $10^{-1}-10^{-19} \mathrm{~cm}^{-3}$. As a matter of fact, Kröger et al. found that without considering interfacial dipoles in their system it is not possible to explain the tunneling mechanism. Therefore, Figs. 11(a) and 11(b) have to be seen as a simplified sketch. In the case of the wide-gap $\mathrm{Al}_{2} \mathrm{O}_{3}$ the interlayer leads to an additional tunneling barrier (see Fig. 11(d)), resulting in an increased drive voltage as we found in our experiments. By inserting the narrow-gap CuPc interlayer the large band bending at the interface may result in an additional tunneling path from the HOMO of the $p$-type organic semiconductor via the LUMO of $\mathrm{CuPc}$ to the LUMO of the $n$-type semiconductor as indicated in Fig. 11(f). The sketch of the energy-

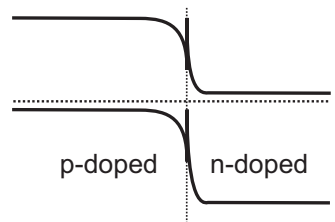

(a.) no external bias

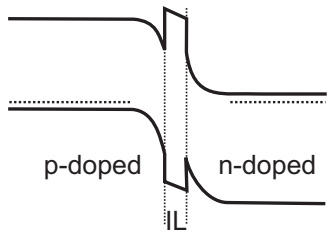

(c.) no external bias, with $\mathrm{Al}_{2} \mathrm{O}_{3}$ IL

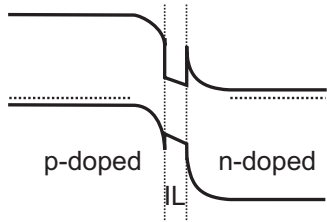

(e.) no external bias, with $\mathrm{CuPc}$ IL (b.) under reverse bias

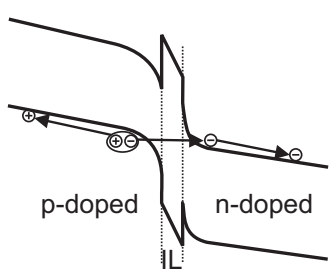

(d.) under reverse bias, with $\mathrm{Al}_{2} \mathrm{O}_{3} \mathrm{IL}$

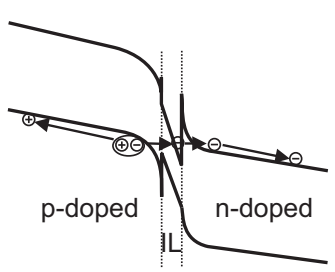
with $\mathrm{CuPc}$ IL

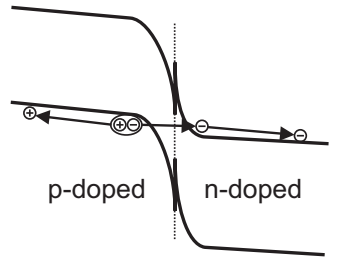

(f.) under reverse bias,

FIG. 11. Simplified model of the energy-level alignment for a CGL device without IL ((a) and (b)), with $\mathrm{Al}_{2} \mathrm{O}_{3}$ IL ((c) and (d)) and with CuPc IL ((e) and (f)): (a), (c), and (e) no external bias, (b), (d), and (f.) under reverse bias. level diagram is not correct in a quantitative sense, but it qualitatively explains the observed charge generation. Further measurements by, e.g., ultraviolet photoelectron spectroscopy (UPS) would be helpful to clarify the exact values of the energy levels but are beyond the scope of this publication.

Furthermore, the tunneling probability of charge carriers through the depletion zone could be further enhanced by the thin CuPc IL within the CGL, because of the formation of an additional interfacial dipole. Lai $e t a l^{36}$ found that there is an interfacial dipole between $\mathrm{CuPc}$ and $\mathrm{F}_{16} \mathrm{CuPc}$. Another important aspect is that $\mathrm{CuPc}$ may introduce additional gap states. Such gap states have been proposed in several reports on enhanced charge carrier injection by inserting CuPc layers between the anode and the organic layer sequence. ${ }^{29-31,33}$ Schöbel found by UPS measurements additional gap states which explained the improved charge carrier injection. ${ }^{34}$ In addition, in other CGL systems gap states have been deliberately introduced for assisting charge carrier injection by inserting an $\mathrm{Li}_{2} \mathrm{O}$ interlayer ${ }^{35}$ or by inserting a combination of a thin LiF/Al layer. ${ }^{5}$ In our case, such gap states could assist electron tunneling from the HOMO of the $p$-doped layer to the LUMO of the $n$-doped layer.

Finally, band-to-band tunneling from the HOMO of $\mathrm{CuPc}$ to the LUMO of CuPc is also likely, if the band bending is large enough. Our experimental findings of the increased current density for the device with the $4 \mathrm{~nm} \mathrm{CuPc}$ IL support these hypotheses explaining the very efficient charge generation.

\section{CONCLUSION}

We showed that a highly stable CGL based on a doped $p$ $n$ junction can be formed by using $\mathrm{MoO}_{3}$ as $p$-type dopant and $\mathrm{Cs}_{3} \mathrm{PO}_{4}$ as novel $n$-type dopant. The investigation of two different classes of interlayer material inserted within the doped junction, on the one hand, the narrow-gap organic material $\mathrm{CuPc}$, and on the other hand, the wide-gap oxidic material $\mathrm{Al}_{2} \mathrm{O}_{3}$, demonstrated that both types of IL help to stabilize the CGL. Furthermore, the voltage consumption of a CGL comprising a thin IL of $\mathrm{CuPc}$ can be further improved and an optimal IL thickness of $8 \mathrm{~nm}$ can be found as a trade-off between minimal operating voltage and maximum voltage stability of the CGL. Luminance-current density-voltage as well as lifetime measurements on stacked green OLEDs confirmed the good functionality and stability of the developed CGL. The lifetime of the stacked device was enhanced by a factor of 3.5 without reduction in efficiency or luminous flux. We discussed the charge generation mechanism of the CGL with different types of IL and proposed a model of the energy-level alignment which describes consistently our experimental findings. From our point of view, the IL is needed to prevent chemical reactions or dopant interdiffusion at the $p-n$ interface leading to a reduced drive voltage and an enhanced stability of the devices. By choosing a proper IL material, e.g., CuPc, the energy-level alignment at the interface can be modified by interfacial dipoles, and gap states can be deliberately introduced leading to an enhanced tunneling current and therefore increased performance of stacked OLEDs. 


\section{ACKNOWLEDGMENTS}

The authors like to thank the OSRAM OLED team as well as Dr. G. Schmid from Siemens AG, Corporate Technology, for the supply of purified caesium phosphate and for valuable discussions. This work was supported by the German Federal Ministry of Education and Research (BMBF) under Contract No. FKZ 13N10474 (TOPAS 2012).

${ }^{1}$ NanoMarkets, OLED Lighting Global Market Forecasts, May 2011, www.nanomarkets.net.

${ }^{2}$ J. Kido, T. Matsumoto, T. Nakada, J. Endo, K. Mori, N. Kawamura, and A. Yokoi, SID Int. Symp. Digest Tech. Papers 34, 979 (2003).

${ }^{3}$ Q. Y. Bao, J. P. Yang, Y. Q. Li, and J. X. Tang, Appl. Phys. Lett. 97, 063303 (2010).

${ }^{4}$ L. Liao, W. Slusarek, T. Hatwar, M. Ricks, and D. Comfort, Adv. Mater. 20, 324 (2008).

${ }^{5}$ T. Chiba, Y.-J. Pu, R. Miyazaki, K. Nakayama, H. Sasabe, and J. Kido, Org. Electron. 12, 710 (2011).

${ }^{6}$ L. S. Liao, K. P. Klubek, and C. W. Tang, Appl. Phys. Lett. 84, 167 (2004).

${ }^{7}$ H. Kanno, N. C. Giebink, Y. Sun, and S. R. Forrest, Appl. Phys. Lett. 89, 023503 (2006).

${ }^{8}$ M. Terai and T. Tsutsui, Appl. Phys. Lett. 90, 083502 (2007).

${ }^{9}$ T. Tsutsui and M. Terai, Appl. Phys. Lett. 84, 440 (2004).

${ }^{10}$ G. Gu, G. Parthasarathy, P. E. Burrows, P. Tian, I. G. Hill, A. Kahn, and S. R. Forrest, J. Appl. Phys. 86, 4067 (1999).

${ }^{11}$ J. Meyer, A. Shu, M. Kroger, and A. Kahn, Appl. Phys. Lett. 96, 133308 (2010).

${ }^{12}$ Q. Wang, J. Ding, Z. Zhang, D. Ma, Y. Cheng, L. Wang, and F. Wang, J. Appl. Phys. 105, 076101 (2009).

${ }^{13}$ M. Kröger, S. Hamwi, J. Meyer, T. Dobbertin, T. Riedl, W. Kowalsky, and H.-H. Johannes, Phys. Rev. B 75, 235321 (2007).

${ }^{14}$ L. S. Liao and K. P. Klubek, Appl. Phys. Lett. 92, 223311 (2008).

${ }^{15}$ T.-W. Lee, T. Noh, B.-K. Choi, M.-S. Kim, D. W. Shin, and J. Kido, Appl. Phys. Lett. 92, 043301 (2008).

${ }^{16}$ J. H. Wemken, R. Krause, T. Mikolajick, and G. Schmid, J. Appl. Phys. 111, 074502 (2012).
${ }^{17}$ R. Krause, Ph.D. dissertation, University of Erlangen-Nuremberg, Germany, 2008.

${ }^{18}$ R. Chance, A. Prock, and R. Silbey, Adv. Chem. Phys. 37, 1 (1978).

${ }^{19}$ N. Danz, R. Waldhäusl, A. Bräuer, and R. Kowarschik, J. Opt. Soc. Am. B 19, 412 (2002).

${ }^{20}$ D. S. Setz, T. D. Schmidt, M. Flämmich, S. Nowy, J. Frischeisen, B. C. Krummacher, T. Dobbertin, K. Heuser, D. Michaelis, N. Danz, W. Brütting, and A. Winnacker, J. Photon. Energy 1, 011006 (2011).

${ }^{21}$ Purchased from Merck KGaA, Darmstadt, Germany.

${ }^{22}$ Z. Shen, P. E. Burrows, V. Bulovic, D. M. McCarty, M. E. Thompson, and S. R. Forrest, Jpn. J. Appl. Phys., Part 2 35, L401 (1996).

${ }^{23}$ V. I. Adamovich, S. R. Cordero, P. I. Djurovich, A. Tamayo, M. E. Thompson, B. W. DAndrade, and S. R. Forrest, Org. Electron. 4, 77 (2003).

${ }^{24}$ J. Staudigel, M. Stößel, F. Steuber, and J. Simmerer, J. Appl. Phys. 86, 3895 (1999)

${ }^{25}$ C.-L. Lin, T.-Y. Cho, C.-H. Chang, and C.-C. Wu, Appl. Phys. Lett. 88, 081114 (2006).

${ }^{26}$ H. Aziz, Z. D. Popovic, and N.-X. Hu, Appl. Phys. Lett. 81, 370 (2002).

${ }^{27}$ J. Meyer, M. Kröger, S. Hamwi, F. Gnam, T. Riedl, W. Kowalsky, and A. Kahn, Appl. Phys. Lett. 96, 193302 (2010).

${ }^{28}$ X. D. Gao, J. Zhou, Z. T. Xie, B. F. Ding, Y. C. Qian, X. M. Ding, and X. Y. Hou, Appl. Phys. Lett. 93, 083304 (2008).

${ }^{29}$ T. Dobbertin, Ph.D. dissertation, University of Braunschweig, Germany, 2005.

${ }^{30}$ S. Tadayyon, H. Grandin, K. Griffiths, P. Norton, H. Aziz, and Z. Popovic, Org. Electron. 5, 157 (2004).

${ }^{31}$ S. Yim, S. Heutz, and T. S. Jones, J. Appl. Phys. 91, 3632 (2002).

${ }^{32}$ G. Jabbour, Y. Kawabe, S. Shaheen, J. Wang, M. Morrell, B. Kippelen, and N. Peyghambarian, Appl. Phys. Lett. 71, 1762 (1997).

${ }^{33}$ G. Parthasarathy, P. E. Burrows, V. Khalfin, V. G. Kozlov, and S. R. Forrest, Appl. Phys. Lett. 72, 2138 (1998).

${ }^{34}$ J. Schöbel, Ph.D. dissertation, University of Braunschweig, Germany, 2001.

${ }^{35}$ H. Kanno, Y. H. N. Okumoto, N. Saito, H. Ishida, H. Takahashi, K. Shibata, and K. Mameno, Jpn. J. Appl. Phys., Part 1 45, 9219 (2006).

${ }^{36}$ S. L. Lai, M. Y. Chan, M. K. Fung, C. S. Lee, and S. T. Lee, J. Appl. Phys. 101, 014509 (2007) 